\title{
Programa Mais Médicos e suas contribuições para a saúde no Brasil: revisão integrativa
}

\author{
José Jeová Mourão Netto, ${ }^{1}$ Antonia Regynara Moreira Rodrigues, ${ }^{2}$ Otávia \\ Cassimiro Aragão, ${ }^{1}$ Natália Frota Goyanna, ${ }^{3}$ Ana Egliny Sabino Cavalcante, ${ }^{1}$ \\ Maria Aparecida Sousa Vasconcelos, ${ }^{4}$ Janice D’Ávila Rodrigues Mendes ${ }^{4}$ \\ e Antonio Edie Brito Mourão ${ }^{4}$
}

Como citar Mourão Netto JJ, Rodrigues ARM, Aragão OC, Goyanna NF, Cavalcante AES, Vasconcelos MAS, et al. Programa Mais Médicos e suas contribuições para a saúde no Brasil: revisão integrativa. Rev Panam Salud Publica. 2018;42:e2. https://doi.org/10.26633/RPSP.2018.2

RESUMO Objetivo. Identificar as contribuições do Programa Mais Médicos (PMM) para a saúde no Brasil.

Método. Revisão integrativa, com busca realizada nas bases de dados SciELO, Lilacs, Medline, Scopus e Web of Science. Foi utilizado o termo de busca "mais médicos" em português, espanhol ("más médicos") e inglês ("more doctors").

Resultados. Foram identificados 1482 artigos, dos quais 35 foram incluídos na revisão. Os estudos revelaram resultados positivos do PMM, com aumento no número de médicos disponíveis, oferta de novas vagas em cursos de medicina, maior disponibilidade de consultas médicas e melhorias na estrutura física e nos processos de trabalho nas unidades básicas de saúde.

Conclusões. O PMM trouxe contribuições significativas para a saúde brasileira, sendo responsável pela mobilização de recursos financeiros para a atenção primária em saúde no país.

Palavras-chave

Programas Nacionais de Saúde; atenção primária à saúde; recursos humanos; saúde pública; Brasil.

No Brasil, a carência de médicos tem limitado o acesso e a cobertura universais à saúde e impulsionado o desenvolvimento de estratégias como o Programa Mais Médicos (PMM), cujo intuito é prover esses profissionais, sobretudo no âmbito da atenção primária à saúde (APS). $\mathrm{O}$ PMM se propõe a qualificar profissionais, investir na infraestrutura das unidades básicas de saúde (UBS) e ampliar a provisão de médicos brasileiros e estrangeiros em municípios com maior

\footnotetext{
Hospital Regional Norte, Sobral (CE), Brasil. Correspondência: José Jeová Mourão Netto, jeovamourao@gmail.com

2 Universidade Estadual do Ceará, Programa de Pós-Graduação em Cuidados Clínicos em
} Enfermagem e Saúde, Fortaleza (CE), Brasil. vulnerabilidade (1, 2). Até 2015, o PMM contava com 18240 médicos (1).

O PMM, entretanto, tem gerado discussão. De um lado, as representações de classe médicas afirmam que o Brasil tem profissionais suficientes para suprir a demanda nacional e cobram melhores condições de trabalho. Já o Ministério da Saúde estima a necessidade de um número maior de médicos diante da atual distribuição de 1,8 profissional por 1000 habitantes, que é inferior a muitos países

\footnotetext{
Prefeitura Municipal de Sobral, Estratégia Trevo de Quatro Folhas, Sobral (CE), Brasil.

Instituto Superior de Teologia Aplicada (INTA), Sobral (CE), Brasil.
}

da Europa e América Latina, trazendo ainda como argumento a grande disparidade na distribuição dos profissionais entre as regiões do país $(3,4)$.

Cabe salientar que, no Brasil, os programas de provimento de profissionais remontam à época da ditadura militar, iniciando com o Projeto Rondon, que objetivava levar estudantes a atuarem em locais de difícil acesso. Posteriormente, surgiu o Programa de Interiorização das Ações de Saúde e Saneamento (1976), o Programa de Interiorização do Sistema Único de Saúde (1993) e o Programa de Interiorização do Trabalho em Saúde (2001), sendo este último uma das estratégias de fortalecimento do Programa 
Saúde da Família (1997), que surgia à época e foi responsável pela ampliação da cobertura assistencial. Finalmente, em 2012, iniciou-se o Programa de Valorização dos Profissionais da Atenção Básica (5). Assim, o PMM não representa a primeira iniciativa governamental no intuito de sanar a problemática da escassez e má distribuição de médicos; no entanto, parece ter sido a mais bem-sucedida.

Diante desse cenário, este estudo objetivou identificar se houve contribuições para a saúde no Brasil a partir da implantação e implementação do PMM no que concerne ao provimento, formação e qualificação de médicos e à estrutura das UBS.

\section{MATERIAIS E MÉTODOS}

Foi realizada uma revisão integrativa da literatura tendo como pergunta norteadora: o PMM contribuiu para a saúde no Brasil? A revisão integrativa compreende cinco etapas: estabelecimento do problema; seleção da amostra; caracterização dos estudos; análise e discussão dos resultados; e apresentação (6). Embora proporcione uma abordagem ampla sobre um objeto, a inclusão de estudos de diferentes delineamentos pode envolver maior complexidade na análise, que, no entanto, pode resultar em maior aprofundamento do objeto de estudo (7).

A busca foi realizada em agosto de 2017 nas bases de dados SciELO,
Medline, Lilacs, Scopus e Web of Science. Foi utilizada para a busca a expressão "mais médicos" em português, espanhol (más médicos) e inglês (more doctors).

As referências dos artigos selecionados foram revisadas manualmente a fim de identificar outras publicações que atendessem a pergunta norteadora e que não haviam sido detectadas pela estratégia de busca. Foram incluídos estudos relacionados ao tema em inglês, português ou espanhol, sem recorte temporal. Excluíram-se livros, monografias, dissertações, teses, textos governamentais, artigos de opinião, editoriais, relatos de experiência, artigos de revisão e textos que não atendessem a pergunta norteadora.

Os textos foram selecionados inicialmente a partir da leitura de seu título e resumo, a fim de verificar a sua consonância com a questão norteadora; quando adequados, foram lidos e analisados na íntegra. Para a organização e a análise, os achados foram dispostos em categorias temáticas.

\section{RESULTADOS}

A busca nas bases e bancos de dados revelou 1482 textos. Após a leitura dos títulos e resumos, foram selecionados 25 artigos. A revisão manual das referências citadas nos textos selecionados revelou mais 50 artigos, dos quais 10 foram incluídos após leitura na íntegra. Assim, 35 artigos compuseram o corpus (figura 1).

FIGURA 1. Fluxograma de seleção dos artigos, 2017

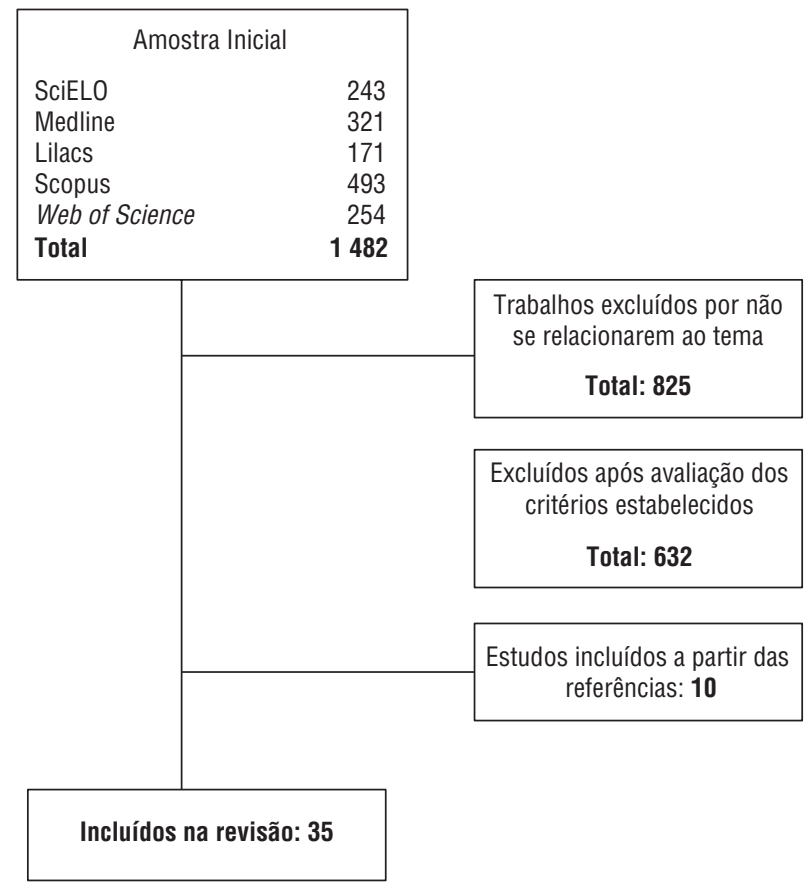

Desses artigos, apenas um foi publicado em revista não editada no Brasil, em língua inglesa (8). Todos os demais correspondem a estudos publicados em periódicos nacionais (9-42).

Predominaram estudos da área de saúde coletiva. Quanto à abrangência, 13 estudos eram nacionais $(8-11,13,18,24$, $25,27,29,35,38,40)$, dois envolveram a região Nordeste $(19,36)$ e um abordou simultaneamente as regiões Norte, Nordeste e Sul (39). Os demais abordaram estados e municípios, sendo desenvolvidos em 13 estados diferentes (12, 14-17, 20-23, 26, 28, 30-34, 37, 41, 42). Quanto à metodologia, a abordagem quantitativa foi utilizada por 21 estudos (8-10, 13-16, $18,19,23,25,27,29-32,35,38,40,41)$, a qualitativa por $12(11,12,20-22,26,28$, $33,34,37,39,42)$ e a quanti-qualitativa, por dois $(17,36)$.

A análise dos artigos revelou cinco categorias temáticas: provimento de médicos, estrutura física das UBS, formação de médicos, inserção dos médicos nos territórios e relação com a população e repercussões da implementação nos territórios. Os textos foram, então, agrupados conforme essas categorias.

\section{Provimento de médicos}

Conforme os estudos analisados, foram contratados pelo PMM médicos de 47 nacionalidades, dos quais 11 150, cerca de $80 \%$, vieram de Cuba (29). A região Nordeste foi a que mais recebeu médicos cooperantes. Nessa região, a razão de médicos por 1000 habitantes passou de 1,23 em 2012 para 1,34 em $2014(19,29)$.

Entre as regiões, a maior incorporação ocorreu na região Norte (52,8\%). Em todo o país, os municípios com menos de 100000 habitantes foram os que mais receberam médicos (9). A proporção de médicos do PMM nos municípios de extrema pobreza foi quase 3 vezes maior do que nas capitais e municípios mais ricos (35).

No período imediatamente anterior ao programa, havia 1200 municípios com escassez de médicos $(21,6 \%$ do total de municípios brasileiros). Já em setembro de 2015, após cerca de 2 anos, esse número caiu para $777(14 \%)$, ressaltando-se que 272 dos municípios com carência não possuíam médicos do $\operatorname{PMM}(24,35)$.

A redução da escassez entre 2013 e 2015 ocorreu em todas as regiões. No fim de 2015 , 73\% dos municípios em todos os estados estavam participando do programa $(24,35)$ e a melhoria do acesso foi 
percebida em muitas realidades $(25,28$, 30). Ressalta-se que os critérios de participação no PMM são definido pelo Ministério da Saúde, que lança editais para que os municípios se inscrevam. Nos municípios de pequeno porte, foi possível ampliar o acesso e alcançar uma cobertura por médicos de quase $100,0 \%$ da população. Contudo, ainda existem diferenças importantes na cobertura, a depender da região (9). Em 2012, havia um total de 6948 equipes, com cobertura de $77,9 \%$ para os municípios inscritos no programa. Em 2015, havia 8038 equipes e uma cobertura de $86,3 \%$ nesses municípios. Nesse período, com o PMM, o crescimento no número de médicos foi menor do que $10 \%$ somente em cinco estados brasileiros: Santa Catarina, São Paulo, Rio de Janeiro, Goiás e Pernambuco $(8,35)$. Sem o programa, 12 estados teriam aumento menor que $10 \%$ (35).

No contexto da saúde indígena, um estudo nacional envolvendo 613 indígenas relatou que $47 \%$ afirmaram que a equipe de saúde que atende a comunidade não incluía médico antes do início do PMM. Isso mostra que, mesmo em caráter emergencial, essa necessidade foi suprida, uma vez que, em 2015, o programa estava em todos os Distritos Sanitários Especiais Indígenas (DSEI) do país, fazendo com que, pela primeira vez, todos contassem com médicos $(35,38)$.

No entanto, mesmo com avanços no provimento, um estudo com 1303 municípios do Nordeste mostrou que a incorporação desses médicos na APS resultou em menor elevação da razão de médicos do que o esperado, característica que se reproduziu em todos os estados da região, repercutindo, ainda, em pequena variação de cobertura das equipes de APS nesses estados (36).

\section{Estrutura física das UBS}

A qualificação da estrutura das UBS configura um importante eixo de atuação do PMM, sendo destinados recursos para a construção de 3496 novas UBS e reforma de outras 3417 (8). Um estudo de abrangência nacional evidenciou que a infraestrutura média das UBS que receberam médicos do PMM é melhor que a das unidades que não os receberam (27), sinalizando que os recursos destinados podem ter influenciado na melhoria dessas unidades. Isso foi confirmado em estudo realizado com supervisores do PMM; os supervisores afirmaram que, além de proporcionar a chegada e a fixação de médicos, o programa contribuiu com a melhoria da estrutura da APS, especialmente nos municípios menores (28). No estado de Mato Grosso, as condições físicas das unidades rurais, onde atuam 50\% dos médicos do PMM, foram consideradas, em sua maioria, inadequadas, e os processos de trabalho foram considerados também inapropriados em $86 \%$ dos relatórios de supervisão, sobretudo pela falta de materiais (28). No Rio Grande do Norte, também identificaram-se problemas na disponibilidade de consultas com especialistas, medicamentos e exames complementares (33).

A partir do disposto, infere-se que o PMM provocou uma movimentação de recursos significativos para a melhoria da estrutura das UBS. Contudo, os estudos que compuseram a revisão não descrevem de forma consistente qual a condição atual das UBS contempladas por esses recursos, o que, diante dos relatos de condições limitadas para as equipes, suscita a necessidade de mais estudos para a compreensão dessas fragilidades.

\section{Formação de médicos}

De 2013 a 2016, foram criados 70 novos cursos de medicina (5 540 novas vagas) em decorrência do PMM. Com isso, o número de vagas de medicina cresceu de 0,83 por 10000 habitantes em 2012 para 1,07 por 10000 habitantes em 2015. Mais de $54 \%$ das novas escolas situam-se nas regiões mais necessitadas desse profissional. Em 2012, havia 1,9 vaga de medicina por 10000 habitantes no Sudeste, 0,9 no Sul, 0,8 no Norte, 0,7 no Centro-Oeste e 0,7 no Nordeste (8). Esses movimentos concorrem para os propósitos do programa, entre os quais está o aumento do número de vagas e a melhor distribuição das vagas no país.

As vagas em residências médicas duplicaram entre 2009 e 2014, com melhor distribuição pelo país. $\mathrm{O}$ aumento mais expressivo foi nas regiões Norte e Nordeste, onde também surgiram residências em especialidades antes inexistentes. Como repercussão do PMM, houve aumento expressivo de vagas de residência em 2014 (5 125) em comparação com 2013 (2546) (40).

Atualmente, há 1277 vagas de residência em Medicina de Família e Comunidade distribuídas por todas as regiões, porém não em todos os estados. Contudo, apesar de terem dobrado entre 2009 e 2014, as vagas nessa especialidade têm ocupação baixa, com taxa de ociosidade em torno de 70\% (40). Essa situação corrobora a percepção de que existe desinteresse pela APS por parte dos médicos.

No estado do Rio de Janeiro, duas novas turmas foram autorizados em cursos de medicina já existentes. Também foram autorizadas 236 novas vagas de graduação, sendo seis delas em instituições públicas e 230 em privadas. Portanto, 98,2\% das 341 vagas autorizadas por meio do PMM estão em cursos privados. Também foram criadas 273 novas vagas de residência, grande parte das quais em municípios do interior (31). Embora isso possa contribuir para uma melhor distribuição desses profissionais no estado, sabe-se que essa estratégia não garante a fixação dos médicos nem sua inserção na APS. Aos integrantes do PMM é oferecida uma formação (especialização em saúde da família). Um estudo mostra que os médicos do PMM consideram a formação como oportunidade que permite a ampliação do conhecimento sobre os princípios e as diretrizes do SUS, o fortalecimento do trabalho das equipes e a melhoria da prática clínica (39). Em um estudo com estudantes e professores de medicina, demonstrou-se que o conhecimento sobre o PMM é incipiente (22).

Outra contribuição do PMM seria a possibilidade de aprendizado a partir da interação entre estudantes de medicina com médicos de outros países, sobretudo cubanos. Essa perspectiva foi pouco abordada pelos estudos. Um deles, porém, relatou que a experiência com os médicos do programa foi importante para o aprendizado sobre relação médico-paciente, trabalho em equipe e aplicação de ações de educação em saúde junto à comunidade (34). No entanto, o mesmo estudo mostrou que as condutas de muitos médicos estrangeiros do PMM estavam em desacordo com protocolos utilizados no Brasil (34).

\section{Repercussões da implementação nos territórios}

Os estudos indicam mudanças significativas nos indicadores de saúde nos municípios que aderiram ao programa, como aumento no número absoluto de consultas, aumento de atendimentos a pessoas com tuberculose e hanseníase, 
aumento das visitas domiciliares de médicos, redução na solicitação de exames e encaminhamentos para especialistas (23) e aumento de consultas de pré-natal (30).

Houve aumento de consultas médicas quando comparados os meses de janeiro de 2013 e janeiro de 2015. Nos municípios participantes do PMM, esse crescimento foi de 33\%; nos demais, de apenas $15 \%$. Embora não seja o único fator, o PMM influiu significativamente para a melhoria desse dado (35).

Houve também melhora no fluxo dos processos nas UBS, pois observou-se que os médicos do PMM encaminham menos para especialistas e para urgências nas capitais (10). O tempo de espera entre o momento que o usuário solicita a consulta e o dia do atendimento foi avaliado como muito bom e bom por $87,3 \%$ dos usuários de equipes do programa, demonstrando mais facilidade de acesso à consulta (18).

Ao se analisar o acesso após a implantação doPMMem 12 municípios, constatouse aumento do número de consultas na população adulta e idosa, assim como maior número de atendimentos de doenças sexualmente transmissíveis/Aids (14). A melhora do acesso pode ser atribuída tanto à presença do profissional como também ao maior tempo com médico disponível na unidade (11). No Ceará, uma pesquisa em seis cidades também identificou melhor acessibilidade a partir do PMM (12). Outro estudo, de abrangência nacional, constatou que as equipes do PMM sustentaram maior produtividade semanal em todos os cenários do estudo, em comparação às equipes que não estão incluídas no programa (10).

Em um contraponto, um estudo mostrou aumento significativo do número de encaminhamentos para os ambulatórios de média complexidade após a implantação do PMM, e ainda redução na porcentagem de acompanhamentos de hipertensos e diabéticos (14). O aumento dos encaminhamentos ambulatoriais poderia ser explicado pela melhoria da cobertura. No entanto, a redução do acompanhamento de hipertensos e diabéticos pode sinalizar uma fragilidade da atenção. Outro estudo também identificou queda no número de consultas entre os menores de 1 ano e nas consultas de criança de 5 a 9 anos, embora tenha evidenciado aumento em outras faixas etárias (23).

Em estudo com 78 entrevistados, concluiu-se que, na ótica dos membros das equipes de saúde da família, o PMM contribui também para a implementação de uma assistência integral (11). O atendimento desses médicos tem sido descrito como de enfoque sistêmico, considerando a saúde como resultante de múltiplos fatores - sociais, ambientais, econômicos e subjetivos (20).

Um estudo confirmou que houve redução da porcentagem de hospitalizações por condições sensíveis à atenção primária em municípios que aderiram ao PMM (de 44,9\% em 2012 para 41,2\% em 2015 , uma diminuição de 8,8\%). Em contrapartida, a taxa permaneceu inalterada durante o período nos municípios sem o programa (8).

Houve melhora em muitos aspectos relativos a APS como impacto do PMM: aumento da cobertura da ESF, maior presença de médicos na APS, aumento de gastos com assistência farmacêutica, adscrição da população aos serviços, além de mudanças ocorridas nas faculdades de medicina, com aumento de residências em Medicina de Família e Comunidade (25). Em outro estudo, a redução de gastrenterites na ordem de 35,0\% foi atribuída à implantação do programa (36).

Em estudo de abrangência nacional, em relação às internações por condições sensíveis à atenção primária, observouse redução em todo o período estudado. Em 2009, ocorreram mais de 2,1 milhões de internações por condições sensíveis à atenção primária em municípios que implantaram o PMM, passando para 1,8 milhão em 2015 (9). No arquipélago de Marajó (estado do Pará), que abrange 16 municípios, houve uma tendência à redução das internações por condições sensíveis à atenção primária em menores de 5 anos de 2013 a 2017. Os resultados indicaram que o PMM foi potencialmente relevante para a redução da morbimortalidade na faixa etária estudada (16). No entanto, a análise de indicadores após a implantação do programa no estado de Pernambuco indicou que não houve alteração na média de internações hospitalares e nem no número de óbitos (41). No estado do Paraná não foram encontradas diferenças quanto aos atributos essenciais da APS na percepção dos profissionais quando comparadas as unidades com e sem o PMM (21).

Um estudo de caráter qualitativo ouviu gestores, supervisores e usuários envolvidos na implementação do PMM em um município do Nordeste. A gestora avaliou o trabalho da médica estrangeira como de qualidade. Uma unanimidade entre os beneficiários foi a avaliação positiva do atendimento médico, descrito como humanizado, de abordagem integral e compromissado (26). O atendimento dos médicos do programa é considerado de qualidade e satisfatório, sendo referido como humanizado, comprometido, de escuta ativa e solidário em parte considerável dos estudos $(11,17$, $18,20,26,28,33,37,38,42$ ).

\section{Inserção dos médicos nos territórios e relação com a população}

Embora o PMM envolva estratégias de forte conotação social, os usuários do sistema de saúde demonstram pouco conhecimento sobre o programa $(12,26,38)$. Por sua vez, a receptividade por parte da população foi relatada como amistosa $(36,38)$. Dois estudos, ambos de abordagem qualitativa, referiram que o atendimento dos médicos estrangeiros do programa tem mais aceitação em comparação ao dos médicos brasileiros $(12,37)$.

Para profissionais e usuários, a diferença do idioma tem representado um empecilho na comunicação, mas não inviabiliza a consulta $(11,12,18,33,37,38)$. Esse aspecto tem sido superado com o emprego de estratégias comunicacionais, como linguagem não verbal, fala pausada e repetida e auxílio de outros profissionais $(11,33)$.

\section{DISCUSSÃO}

A escassez e a má distribuição de médicos é um problema enfrentado em vários países no mundo, com impacto direto na universalização do acesso à saúde (43). Diante dessa realidade, muitos países procuram estratégias para ampliar a cobertura dos serviços, principalmente em áreas vulneráveis, atraindo profissionais de saúde para essas regiões $(44,45)$.

Na Austrália, há quase duas décadas, médicos australianos formados no exterior e estrangeiros formados na Austrália são enviados para regiões de difícil acesso. Eles são contratados por até 10 anos, com remuneração proporcional à distância entre a região geográfica de atuação e o centro urbano mais próximo (46).

Em 2003, a Venezuela iniciou o Programa Misión Barrio Adentro, trazendo de Cuba mais de 20 mil médicos para 
trabalhar na atenção primária. O mesmo programa promoveu o retorno de 3328 estudantes venezuelanos formados em medicina geral em Cuba (47). No México, outra alternativa foi utilizada para fixar os médicos em regiões remotas: o serviço social obrigatório para profissionais de saúde. Nessa estratégia, os profissionais, antes de receberem o diploma, devem cumprir um tempo de serviço obrigatório em estabelecimentos de saúde localizados em região de difícil acesso e de baixo desenvolvimento socioeconômico, sendo uma iniciativa adotada em muitos países da América Latina (48). No Brasil, das estratégias utilizadas para melhorar o provimento de médicos, sobretudo na atenção primária (5), a de maior êxito foi o PMM (43).

Como mostraram os estudos analisados, o PMM contribui para a melhoria no provimento de médicos no Brasil, sobretudo nas áreas mais vulneráveis. Contribuiu também para uma distribuição geoespacial mais equânime, embora a má distribuição de médicos ainda represente um problema.

O PMM promoveu o direcionamento de recursos financeiros para melhoria da estrutura das UBS. Entretanto, alguns estudos sinalizam fragilidades estruturais que têm interferido no trabalho das esquipes, indicando que pode haver um descompasso entre os investimentos destinados e a real estrutura encontrada pelas equipes do programa. Além disso, também se observou que as UBS em piores condições têm menores chances de participar do PMM, o que enfraquece a potencialidade de redução de iniquidades no acesso aos recursos em saúde (13). Finalmente, um estudo realizado em 32 municípios constatou que, embora o PMM tenha proporcionado a ampliação do acesso à consulta médica, ainda persistem problemas como o acesso limitado a exames complementares (11) e a falta de materiais básicos nas UBS, como medicamentos (28).

Embora tenha emergido em apenas um estudo (34), a questão da adequação dos médicos estrangeiros aos protocolos nacionais é fundamental para o desenvolvimento do trabalho no âmbito da APS brasileira. Essa questão precisa ser melhor elucidada.

Poucos foram os estudos que avaliaram o impacto do PMM na formação dos profissionais. Mesmo diante disso, é possível constatar que o programa impulsionou a expansão do número de vagas de graduação e residência médica, bem como a melhor distribuição dessas vagas entre as regiões mais vulneráveis do país. No caso da ampliação das vagas de graduação, essas ocorreram, sobretudo, pela abertura em instituições privadas. Finalmente, algumas experiências apontam que o PMM não repercutiu como esperado ou repercutiu de forma

\section{REFERÊNCIAS}

1. Brasil. Ministério da Saúde. Programa Mais Médicos: 2 anos. Brasília: Ministério da Saúde; 2015. Disponível em: http:/ / portalsaude.saude.gov.br/images/pdf/2015/ agosto/03/livro-maismedicos-2015.pdf Acessado em 12 de dezembro de 2017.

2. Brasil. Lei 12 871/2013. Disponível em: http:/ / www.planalto.gov.br/ccivil_03/_ ato2011-2014/2013/Lei/L12871.htm Acessado em 30 de agosto de 2017.

3. Brasil. Ministério da Saúde (MS). Rede Interagencial de Informação para a Saúde (RIPSA). Indicadores e Dados Básicos - Brasil 2012 IDB-2012. Brasília: MS; 2013.

4. Pereira LL, Santos LMP, Santos W, Oliveira A, Rattner D. Mais Médicos Program: provision of medical doctors in rural, remote and socially vulnerable areas of Brazil, 2013-2014. Rural Remote Health. 2016;16(1):3616.

5. Maciel Filho, R. Estratégias para Distribuição e Fixação de Médicos em Sistemas Nacionais de Saúde [tese]. Rio de Janeiro: Universidade Estadual do Rio de Janeiro; 2007.

6. Ganong LH. Integrative reviews of nursing research. Res Nurs Health. 1987;10(1):1-11.
7. Mendes KDS, Silveira RCCP, Galvão CM. Revisão integrativa: método de pesquisa para a incorporação de evidências na saúde e na enfermagem. Texto Contexto Enferm. 2008;17(4):758-64.

8. Santos LMP, Oliveira A, Trindade JS, Barreto ICHC, Palmeira PA, Comes Y, et al. Implementation research: towards universal health coverage with more doctors in Brazil. Bull World Health Organ. 2017;95(2):103-12.

9. Miranda GMD, Mendes ACG, Silva ALA, Santos Neto PM. A ampliação das equipes de saúde da família e o Programa Mais Médicos nos municípios brasileiros. Trab Educ Saúde. 2017;15(1):131-45.

10. Lima RTS, Fernandes TG, Balieiro AAS, Costa FS, Schramm JMA, Schweickardt JC, et al. A Atenção Básica no Brasil e o Programa Mais Médicos: uma análise de indicadores de produção. Cienc Saude Coletiva. 2016;21(9):2685-96.

11. Comes Y, Trindade JS, Pessoa VM, Barreto ICHC, Shimizu HE, Dewes D, et al. A implementação do Programa Mais Médicos e a integralidade nas práticas da Estratégia Saúde da Família. Cienc Saude Coletiva. 2016;21(9):2729-38. limitada, o que pode estar associado a fatores como dificuldades na organização dos arranjos políticos necessários para essa implantação, disputas de poder partidário (28) ou inserção de áreas não prioritárias no programa.

Cabe ressaltar, como limitação do presente estudo, a impossibilidade de análise mais aprofundada das repercussões do PMM em questões como programas de residência médica e estrutura física das UBS. Essa análise foi limitada pelo pequeno número de estudos disponíveis na literatura e pode ser foco de futuras pesquisas.

Em conclusão, o PMM contribuiu de forma significativa para a saúde brasileira, uma vez que reduziu a escassez de médicos na atenção primária à saúde, impulsionou a expansão do número de vagas de graduação e residência em medicina e foi responsável pela mobilização de recursos financeiros para melhorar a estrutura das unidades básicas de saúde.

Conflitos de interesse. Nada declarado pelos autores.

Declaração. As opiniões expressas no manuscrito são de responsabilidade exclusiva dos autores e não refletem necessariamente a opinião ou política da RPSP/PAJPH ou da Organização Panamericana de Saúde (OPAS).
12. Melo CF, Baião DC, Costa MC. A percepção dos usuários cearenses sobre o Programa Mais Médicos. Gest Soc. 2016;10(26):1302-12.

13. Giovanella L, Mendonça MHM, Fausto MCR, Almeida PF, Bousquat A, Lima JG, et al. A provisão emergencial de médicos pelo Programa Mais Médicos e a qualidade da estrutura das Unidades Básicas de Saúde. Cienc Saude Coletiva. 2016; 21(9):2697-08.

14. Silva BP, Stockmann D, Souza LD, Henna E, Rocha MCP, Junqueira FM. Ampliação do acesso à saúde na região mais vulnerável do estado de São Paulo, Brasil: reflexo do Programa Mais Médicos? Cienc Saude Coletiva. 2016;21(9):2899-906.

15. Miranda AS, Melo DA. Análise comparativa sobre a implantação do Programa Mais Médicos em agregados de municípios do Rio Grande do Sul, Brasil. Cienc Saude Coletiva. 2016;21(9):2837-48.

16. Carneiro VB, Oliveira PTR, Bastos MSCB, Alvarenga EC. Avaliação da mortalidade e internações por condição sensível à atenção primária em menores de 5 anos, antes e durante o Programa Mais Médicos, 
no Marajó-Pará-Brasil. Saúde Redes. 2016;2(4):360-71.

17. Santos MAM, Souza EG, Cardoso JC. Avaliação da qualidade da Estratégia Saúde da Família e do Programa Mais Médicos na área rural de Porto Velho, Rondônia. Gest Soc. 2016;10(26):1327-46.

18. Comes Y, Trindade JS, Shimizu HE, Hamann EM, Bargioni F, Ramirez L, et al. Avaliação da satisfação dos usuários e da responsividade dos serviços em municípios inscritos no Programa Mais Médicos. Cienc Saude Coletiva. 2016;21(9):2749-59.

19. Nogueira PTA, Bezerra AFB, Leite AFB, Carvalho IMS, Gonçalves RF, Brito-Silva KS. Características da distribuição de profissionais do Programa Mais Médicos nos estados do Nordeste, Brasil. Cienc Saude Coletiva. 2016;21(9):2889-98.

20. Guimarães CA, Soares NV, Dorneles JP, Kreuning EB. Pregnant women conception about prenatal care performed by professional of "More Doctors" program. Cinergis. 2016;18(1):25-8.

21. Carrer A, Toso BRGO, Guimarães ATB, Conterno JR, Minosso KC. Efetividade da Estratégia Saúde da Família em unidades com e sem Programa Mais Médicos em município no oeste do Paraná, Brasil. Cienc Saude Coletiva. 2016;21(9):2849-60.

22. Villa Real GGO, Succi GM, Montalli VAM, Succi RCM. Estudantes e Professores da Área da Saúde Conhecem o Programa Mais Médicos? Rev Bras Educ Med. 2017;41(1):110-6.

23. Alencar APA, Xavier SPL, Laurentino PAS, Lira PF, Nascimento VB, Carneiro N, et al. Impacto do Programa Mais Médicos na Atenção Básica de um município do Sertão Central Nordestino. Gest Soc. 2016;10(26):1290-301.

24. Girardi SN, Stralen ACS, Cella JN, Wan DML, Carvalho CL, Faria EO. Impacto do Programa Mais Médicos na redução da escassez de médicos em Atenção Primária à Saúde. Cienc Saude Coletiva. 2016;21(9):2675-84.

25. Mendonça CS, Diercks MS, Kopittke L. Strengthening Primary Health Care in the municipalities in the Metropolitan Region of Porto Alegre, Brazil, after the introduction of the Mais Médicos (More Doctors) Program: an inter-municipal comparison. Cienc Saude Coletiva. 2016;21(9):2871-8.

26. Silva RJ, Sousa D. O Programa Mais Médico na Perspectiva dos atores sociais responsáveis por sua implantação e dos beneficiários no Município de Boqueirão, PB. Reunir. 2015;5(3):59-75.

27. Soares NJJ, Machado MH, Alves CB. O Programa Mais Médicos, a infraestrutura das Unidades Básicas de Saúde e o Índice de Desenvolvimento Humano Municipal. Cienc Saude Coletiva. 2016;21(9):2709-18.

28. Mota RG, Barros NF. O Programa Mais Médicos no Estado de Mato Grosso, Brasil: uma análise de implementação. Cienc Saude Coletiva. 2016;21(9):2879-88.

29. Oliveira JPA, Sanchez MN, Santos LMP. O Programa Mais Médicos: provimento de médicos em municípios brasileiros prioritários entre 2013 e 2014. Cienc Saude Coletiva. 2016;21(9):2719-27.

30. Vargas AFM, Campos MM, Vargas DS. O risco dos extremos: uma análise da implantação do programa Mais Médicos em um contexto de volatilidade orçamentária. Gest Soc. 2016;10(26):1313-26.

31. Silva Junior AG, Andrade HS, Alexandre GC, Alves MGM, Costa BR. Oferta de vagas de graduação e residência médicas no Estado do Rio de Janeiro a partir do Programa Mais Médicos (2013-2015). Gest Soc. 2016;10(26):1347-59.

32. Mendonça FF, Mattos LFA, Oliveira EBD, Domingos CM, Okamura CT, Carvalho BG, et al. Participação dos municípios de pequeno porte no Projeto Mais Médicos para o Brasil na macrorregião norte do Paraná. Cienc Saude Coletiva. 2016;21(9):2907-15.

33. Silva TRB, Silva JV, Pontes AGV, Cunha ATR. Percepção de usuários sobre o Programa Mais Médicos no município de Mossoró, Brasil. Cienc Saude Coletiva. 2016;21(9):2861-69.

34. Sena IS, Guerreiro LC, Ribeiro AC, Morais LSS, Nazina MTST, Santos BEF. Percepções de estudantes de medicina sobre a experiência de aprendizado na comunidade dentro do Programa Mais Médicos: análise de um grupo focal. Tempus Actas Saude Colet. 2015;9(4):91-5.

35. Pinto HA, Oliveira FP, Santana JSS, Santos FOS, Araujo SQ, Figueiredo AM et al. Programa Mais Médicos: avaliando a implantação do Eixo Provimento de 2013 a 2015. Interface (Botucatu). 2017; 21(Supl.1):1087-101.

36. Gonçalves RF, Sousa IMC, Tanaka OY, Santos CR, Brito-Silva K, Santos LX, et al. Programa Mais Médicos no Nordeste: avaliação das internações por condições sensíveis à Atenção Primária à Saúde. Cienc Saude Coletiva. 2016;21(9):2815-24.

37. Carrapato JFL, Silva RVC, Rotondaro EC, Placideli N. Programa Mais Médicos: percepção dos usuários e dos profissionais do SUS. Em Pauta. 2016;38(14):280-93.

38. Fontão MAB, Pereira EL. Projeto Mais Médicos na saúde indígena: reflexões a partir de uma pesquisa de opinião. Interface (Botucatu). 2017;21(suppl.1): 1169-80.
39. Thumé E, Wachs LS, Soares MU, Cubas MR, Fassa MEG, Tomasi E, et al. Reflexões dos médicos sobre o processo pessoal de aprendizagem e os significados da especialização à distância em saúde da família. Cienc Saude Coletiva. 2016;21(9):2807-14.

40. Alessio MM, Sousa MF. Regulação da formação de especialistas: inter-relações com o Programa Mais Médicos. Physis. 2016;26(2):633-67.

41. Silva MN, Freitas MAL. Basic health in Pernambuco: before and after the More Medical Program. Rev Soci Humanas. 2017;30(1):10-20.

42. Lima RCGS, Gripa DW, Prospero ENS, Ros MA. Academic tutorship of the Mais Médicos para o Brasil (More Doctors to Brazil) Project in Santa Catarina: the ethical-political viewpoint. Cienc Saude Coletiva. 2016;21(9):2797-805.

43. Oliveira FP, Vanni T, Pinto HA, Santos JTR, Figueiredo AM, Araújo SQ, et al. Um programa brasileiro em uma perspectiva internacional. Interface (Botucatu). 2015;19(54):623-34

44. Chopra M, Munro S, Lavis JN, Vist G, Bennett $S$. Effects of policy options for human resources for health: an analysis of systematic reviews. Lancet. 2008;371(9613): 668-74.

45. Buykx P, Humphreys J, Wakerman J, Pashen D. Systematic review of effective retention incentives for health workers in rural and remote areas: towards evidencebased policy. Aust J Rural Health. 2010;18(3):102-9.

46. Australian Government, Department of Health. Review of the Australian Government Health Workforce Programs. Canberra: Department of Health; 2013. Disponível em: http://www.health.gov. $\mathrm{au} /$ internet/main/publishing.nsf/content/review-australian-government-health-workforce-programs Acessado em janeiro de 2018

47. Backer LC, Molina A. Cuba and the construction of alternative global trade systems: ALBA and Free Trade in the Americas. J Int Econ L. 2010;31(3):37-8.

48. Maciel Filho R. Estratégias para a distribuição e fixação de médicos em Sistemas Nacionais de Saúde: o caso brasileiro [tese]. Rio de Janeiro: Universidade do Estado do Rio de Janeiro; 2007.

Manuscrito recebido em 23 de maio de 2017. Aceito em versão revisada em 6 de outubro de 2017. 
ABSTRACT Objective. To identify the contributions of the Mais Médicos Program (PMM) for health care in Brazil.

Method. An integrative review was performed in the following databases: SciELO,

Contributions of the Mais Médicos (More Doctors) physician recruitment program for health care in Brazil: an integrative review

Keywords National Health Programs; primary health care; human resources; public health; Brazil.

RESUMEN Objetivo. Identificar las contribuciones del Programa Más Médicos (PMM) para la salud en Brasil.

Método. Se realizó una revisión integrativa en las bases de datos SciELO, LILACS,

EI Programa Más Médicos y sus contribuciones a la salud en Brasil: revisión integrativa

Palabras clave
Medline, Scopus y Web of Science. Utilizamos el término de búsqueda "mais médicos" en portugués, en español ("más médicos") y en inglés ("more doctors").

Resultados. Se identificaron 1482 artículos, 35 de los cuales fueron incluidos en la revisión. Los estudios revelaron resultados positivos del PMM, con aumento en el número de médicos disponibles, oferta de nuevos cupos en cursos de medicina, mayor disponibilidad de consultas médicas, y mejoras en la estructura física y en los procesos de trabajo en las unidades básicas de salud.

Conclusiones. El PMM trajo contribuciones significativas para la salud brasileña, siendo responsable por la movilización de recursos financieros para la atención primaria en salud en el país.

Programas nacionales de salud; atención primaria de salud; recursos humanos; salud pública; Brasil. 